\title{
Systematic study of the chiral magnetic effect with the AVFD model at LHC energies
}

\author{
Panos Christakoglou $^{\mathrm{a}}$, Shi Qiu ${ }^{\mathrm{b}}$, Joey $\operatorname{Staa}^{\mathrm{c}}$ \\ Nikhef, Amsterdam, The Netherlands
}

Received: 7 June 2021 / Accepted: 26 July 2021 / Published online: 10 August 2021

(C) The Author(s) 2021

\begin{abstract}
We present a systematic study of the correlators used experimentally to probe the Chiral Magnetic Effect (CME) using the Anomalous Viscous Fluid Dynamics (AVFD) model in $\mathrm{Pb}-\mathrm{Pb}$ and $\mathrm{Xe}-\mathrm{Xe}$ collisions at $\mathrm{LHC}$ energies. We find a parametrization that describes the dependence of these correlators on the value of the axial current density $\left(n_{5} / \mathrm{s}\right)$, which dictates the CME signal, and on the parameter that governs the background in these measurements i.e., the percentage of local charge conservation (LCC) within an event. This allows to deduce the values of $n_{5} / \mathrm{s}$ and the LCC percentage that provide a quantitative description of the centrality dependence of the experimental measurements. We find that the results in $\mathrm{Xe}-\mathrm{Xe}$ collisions at $\sqrt{s_{\mathrm{NN}}}=5.44 \mathrm{TeV}$ are consistent with a background only scenario. On the other hand, the model needs a significant non-zero value of $n_{5} / \mathrm{s}$ to match the measurements in $\mathrm{Pb}-\mathrm{Pb}$ collisions at $\sqrt{s_{\mathrm{NN}}}=5.02 \mathrm{TeV}$.
\end{abstract}

\section{Introduction}

Collisions between heavy ions accelerated at ultra-relativistic energies provide the necessary conditions to form a deconfined state of matter, the Quark Gluon Plasma [1]. In this phase, the fundamental constituents of quantum chromodynamics (QCD), the quarks and gluons, are not anymore confined inside their usual hadronic bags. The transition to a QGP from normal hadronic matter is expected to take place at a temperature of about $155 \mathrm{MeV}$, and an energy density of about $0.5 \mathrm{GeV} / \mathrm{fm}^{3}$, according to lattice QCD calculations [2-4]. These conditions can be reached in collisions between $\mathrm{Pb}$ ions at the Large Hadron Collider (LHC) [5-7].

Heavy ion collisions also provide the possibility to study novel QCD phenomena that are otherwise not accessible

\footnotetext{
a e-mail: Panos.Christakoglou@ nikhef.nl (corresponding author)

b e-mail: Shi.Qiu@nikhef.nl

c e-mail: Joey.Staa@nikhef.nl
}

experimentally. One characteristic example is related to local parity $(\mathrm{P})$ as well as charge conjugation and parity (CP) symmetry violation in strong interactions. The possibility to observe parity violation in the strong interaction using relativistic heavy-ion collisions has been discussed in [8-10] and was further reviewed in [11-19]. In QCD, this symmetry violation originates from the interaction between the chiral fermions of the theory and topologically non-trivial gluonic fields that induce net-chirality. In the presence of a strong magnetic field, such as the one created in peripheral heavy ion collisions with a magnitude of around $10^{15} \mathrm{~T}$ [20-22], these interactions lead to an asymmetry between left and right-handed quarks. The generated net-chirality, in turns, leads to an excess of positively and negatively charged particles moving in opposite directions relative to the system's symmetry plane. This introduces an electromagnetic current and the creation of an electric dipole moment of QCD matter. The experimental search for these effects has intensified recently, following the realisation that the subsequent creation of charged hadrons results in an experimentally accessible magnitude of charge separation along the direction of this magnetic field, and perpendicular to the symmetry plane. This phenomenon is called the Chiral Magnetic Effect (CME) [23] and its existence was recently reported in semi-metals like zirconium pentatelluride $\left(\mathrm{ZrT} \mathrm{e}_{5}\right)$ [24].

Early enough it was realised that a way to probe these effects is to rely on measuring two-particle azimuthal correlations relative to the reaction plane $\left(\Psi_{\mathrm{RP}}\right)$ [25], the plane defined by the impact parameter and the beam axis. Since then, intensive experimental efforts have been made to identify unambiguously signals of the CME. The first measurements using this approach were reported by the STAR Collaboration in $\mathrm{Au}-\mathrm{Au}$ collisions at $\sqrt{s_{\mathrm{NN}}}=0.2 \mathrm{TeV}[26,27]$ and were consistent with initial expectations for a charge separation relative to the reaction plane due to the CME. Soon after, the first results from the $\mathrm{LHC}$ in $\mathrm{Pb}-\mathrm{Pb}$ collisions at $\sqrt{s_{\mathrm{NN}}}=2.76 \mathrm{TeV}$ were reported and showed a quantita- 
tively similar effect [28]. This agreement between the results is up until this moment hard to comprehend considering the differences in the centre-of-mass energy and consequently in the multiplicity density [29]. In addition, the magnetic field and the way it evolves is, in principle, different between the two energies [20-22]. Overall, this agreement hinted at the dominant role of background effects in both measurements [30]. These background effects were, in parallel, identified as coming from local charge conservation coupled to the anisotropic expansion of the system in non-central collisions $[31,32]$. The field turned its focus to finding a way to constrain and quantify the background and the CME contribution to such measurements.

In Ref. [33], the ALICE Collaboration presented the first ever upper limit of 26-33\% at $95 \%$ confidence level for the CME contribution, using an Event Shape Engineering (ESE) technique [34]. At the same time, the CMS Collaboration reported an upper limit of $7 \%$ at $95 \%$ confidence level using a similar procedure, albeit without a quantification of the possible dependence of these results on the elliptic flow value [35]. In parallel, new measurements of the STAR Collaboration in Au-Au collisions at a centre-ofmass energy $\sqrt{s_{\mathrm{NN}}}=200 \mathrm{GeV}$ [36-38] could not exclude a small contribution of the CME signal to these measurements. Finally, results obtained from the analysis of data collected from the beam energy scan at $\sqrt{s_{\mathrm{NN}}}=7.7,11.5,19.6,27$, 39 and $62.4 \mathrm{GeV}$ [38] were still qualitatively consistent with expectations from parity violating effects in heavy ion collisions. To study background effects the CMS [39] and the STAR [40] collaborations studied charge dependent correlations in both $\mathrm{p}-\mathrm{Pb}$ collisions at $\sqrt{s_{\mathrm{NN}}}=5.02 \mathrm{TeV}$ and in $\mathrm{p}-\mathrm{Au}$ and $\mathrm{d}-\mathrm{Au}$ collisions at $\sqrt{s_{\mathrm{NN}}}=0.2 \mathrm{TeV}$, respectively. Both results illustrate that these correlations are similar to those measured in heavy-ion collisions. The authors concluded that these findings could have important implications for the interpretation of the heavy-ion data since it is expected that the results in these "small" systems are dominated by background effects. However these latter studies are lacking a quantitative estimate of the reaction plane independent background [26,27] and therefore could not be used to extract a definite conclusion. Finally, the ALICE Collaboration recently reported their updated upper limits of $15-18 \%$ at $95 \%$ confidence level for the centrality interval $0-40 \%$ by studying charge dependent correlations relative to the third order symmetry plane [41]. Overall, the extraction of the CME signal has been exceptionally challenging.

In this article we follow a different approach by performing a systematic study of the correlators used in CME searches for $\mathrm{Pb}-\mathrm{Pb}$ and $\mathrm{Xe}-\mathrm{Xe}$ collisions at $\sqrt{s_{\mathrm{NN}}}=$ $5.02 \mathrm{TeV}$ (for Pb ions) $[28,41]$ and at $\sqrt{s_{\mathrm{NN}}}=5.44 \mathrm{TeV}$ (for Xe ions) [42] with the Anomalous-Viscous Fluid Dynamics (AVFD) framework [43-45]. This is a state-of-the-art model that describes the initial state of the collision using a Glauber prescription, and accounts for the development of the early stage electromagnetic fields as well as for the propagation of anomalous fermion currents. The expanding medium is treated after $0.6 \mathrm{fm} / c$ via a $2+1$ dimensional viscous hydrodynamics (VISH2+1) code with value of shear and bulk viscosities over entropy density of $\eta / s=0.08$ and $\zeta / s=0$.Beyond a decoupling energy density of $\epsilon=0.18 \mathrm{fm} / c^{3}$ the system is described by a hadron cascade model (UrQMD) [46]. The analysis is performed in the same kinematic ranges as the experimental measurements for primary charged particles that have a pseudorapidity of $|\eta|<0.8$ and transverse momentum of $0.2<p_{\mathrm{T}}<5 \mathrm{GeV} / c$. The goal of this study is to extract the relevant values that govern the CME signal and the background in the AVFD model that will allow for a quantitative description of the centrality dependence of the charged dependent correlations measured in various colliding systems and energies at the LHC.

The article is organised as follows: Sect. 2 presents the main observables, followed by a discussion on how the model is calibrated in Sect. 3. The main results are presented in Sect. 4 . The article concludes with a summary.

\section{Experimental observables}

A way to probe the parity violating effects is by introducing P-odd coefficients $a_{n, \alpha}$ in the Fourier series frequently used in studies of azimuthal anisotropy [47]. This leads to the expression

$$
\frac{d N}{d \varphi} \approx 1+2 \sum_{n}\left[v_{n} \cos \left[n\left(\varphi-\Psi_{n}\right)\right]+a_{n} \sin \left[n\left(\varphi-\Psi_{n}\right)\right]\right]
$$

where $N$ is the number of particles, $\varphi$ is the azimuthal angle of the particle and $v_{n}$ are the corresponding flow coefficients ( $v_{1}$ : directed flow, $v_{2}$ : elliptic flow, $v_{3}$ : triangular flow etc.). The n-th order symmetry plane of the system, $\Psi_{n}$, is introduced to take into account that the overlap region of the colliding nuclei exhibits an irregular shape [48-52]. This originates from the initial density profile of nucleons participating in the collision, which is not isotropic and differs from one event to the other. In case of a smooth distribution of matter produced in the overlap zone, the angle $\Psi_{n}$ coincides with that of the reaction plane, $\Psi_{\mathrm{RP}}$. In Eq. $1, a_{1}$ is the leading order P-odd term that reflects the magnitude, while higher harmonics (i.e. $a_{2}$ and above) represent the specific shape of the CME signal.

In Ref. [25], Voloshin proposed that the leading order Podd coefficient can be probed through the study of chargedependent two-particle correlations relative to the reaction plane $\Psi_{\mathrm{RP}}$. In particular, the expression discussed is of the form $\left\langle\cos \left(\varphi_{\alpha}+\varphi_{\beta}-2 \Psi_{\mathrm{RP}}\right)\right\rangle$, where $\alpha$ and $\beta$ denote parti- 
cles with the same or opposite charge. This expression can probe correlations between the leading P-odd terms for different charge combinations $\left\langle a_{1, \alpha} a_{1, \beta}\right\rangle$. This can be seen if one expands the correlator using Eq. 1 according to

$$
\begin{aligned}
& \left\langle\cos \left(\varphi_{\alpha}+\varphi_{\beta}-2 \Psi_{\mathrm{RP}}\right)\right\rangle \\
& =\left\langle\cos \left[\left(\varphi_{\alpha}-\Psi_{\mathrm{RP}}\right)+\left(\varphi_{\beta}-\Psi_{\mathrm{RP}}\right)\right]\right\rangle \\
& =\left\langle\cos \left(\Delta \varphi_{\alpha}+\Delta \varphi_{\beta}\right)\right\rangle \\
& =\left\langle\cos \Delta \varphi_{\alpha} \cos \Delta \varphi_{\beta}\right\rangle-\left\langle\sin \Delta \varphi_{\alpha} \sin \Delta \varphi_{\beta}\right\rangle \\
& =\left\langle v_{1, \alpha} v_{1, \beta}\right\rangle+\mathrm{B}_{\text {in }}-\left\langle a_{1, \alpha} a_{1, \beta}\right\rangle-\mathrm{B}_{\text {out }},
\end{aligned}
$$

where $\mathrm{B}_{\text {in }}$ and $\mathrm{B}_{\text {out }}$ represent the parity-conserving correlations projected onto the in- and out-of-plane directions [25]. The terms $\left\langle\cos \Delta \varphi_{\alpha} \cos \Delta \varphi_{\beta}\right\rangle$ and $\left\langle\sin \Delta \varphi_{\alpha} \sin \Delta \varphi_{\beta}\right\rangle$ in Eq. 2 quantify the correlations with respect to the in- and out-ofplane directions, respectively. The term $\left\langle v_{1, \alpha} v_{1, \beta}\right\rangle$, i.e. the product of the first order Fourier harmonics or directed flow, is expected to have negligible charge dependence in the midrapidity region [53]. In addition, for a symmetric collision system the average directed flow at mid-rapidity is zero.

A generalised form of Eq. 2, describing also higher harmonics, is given by the mixed-harmonics correlations and reads

$\gamma_{\mathrm{m}, \mathrm{n}}=\left\langle\cos \left(\mathrm{m} \varphi_{\alpha}+\mathrm{n} \varphi_{\beta}-(\mathrm{m}+\mathrm{n}) \Psi_{|\mathrm{m}+\mathrm{n}|}\right)\right\rangle$,

where $\mathrm{m}$ and $\mathrm{n}$ are integers. Setting $\mathrm{m}=1$ and $\mathrm{n}=1$ (i.e. $\gamma_{1,1}$ ) leads to Eq. 2.

In order to independently evaluate the contributions from correlations in- and out-of-plane, one can also measure a two-particle correlator of the form

$$
\begin{aligned}
& \left\langle\cos \left(\varphi_{\alpha}-\varphi_{\beta}\right)\right\rangle \\
& =\left\langle\cos \left[\left(\varphi_{\alpha}-\Psi_{\mathrm{RP}}\right)-\left(\varphi_{\beta}-\Psi_{\mathrm{RP}}\right)\right]\right\rangle \\
& =\left\langle\cos \left(\Delta \varphi_{\alpha}-\Delta \varphi_{\beta}\right)\right\rangle \\
& =\left\langle\cos \Delta \varphi_{\alpha} \cos \Delta \varphi_{\beta}\right\rangle+\left\langle\sin \Delta \varphi_{\alpha} \sin \Delta \varphi_{\beta}\right\rangle \\
& =\left\langle v_{1, \alpha} v_{1, \beta}\right\rangle+\mathrm{B}_{\text {in }}+\left\langle a_{1, \alpha} a_{1, \beta}\right\rangle+\mathrm{B}_{\text {out }},
\end{aligned}
$$

This provides access to the two-particle correlations without any dependence on the symmetry plane angle which can be generalised according to

$\delta_{\mathrm{m}}=\left\langle\cos \left[\mathrm{m}\left(\varphi_{\alpha}-\varphi_{\beta}\right)\right]\right\rangle$.

This correlator, owing to its construction, is affected if not dominated by background contributions. Charge-dependent results for $\delta_{1}$, together with the relevant measurements of $\gamma_{1,1}$ were first reported in Ref. [28] and made it possible to separately quantify the magnitude of correlations in- and out-of-plane.

\section{Model calibration and parametrisation}

The goal of this study is to extract the values that control the CME signal and the background in the AVFD model that will allow for a quantitative simultaneous description of the centrality dependence of the charged dependent correlations, i.e. $\Delta \delta_{1}$ and $\Delta \gamma_{1,1}$ measured in $\mathrm{Pb}-\mathrm{Pb}$ collisions at $\sqrt{s_{\mathrm{NN}}}=5.02 \mathrm{TeV}[28,41]$ and in $\mathrm{Xe}-\mathrm{Xe}$ collisions at $\sqrt{s_{\mathrm{NN}}}=5.44 \mathrm{TeV}$ [42]. Here, $\Delta \delta_{1}$ and $\Delta \gamma_{1,1}$ denote the difference of $\delta_{1}$ and $\gamma_{1,1}$ between opposite- and same-sign pairs. Within the AVFD framework, the CME signal is controlled by the axial current density $n_{5} / \mathrm{s}$ which dictates the imbalance between right- and left-handed fermions induced in the initial stage of each event. The parameter that governs the background is represented by the percentage of local charge conservation (LCC) within an event. This number can be considered as the amount of positive and negative charged partners emitted from the same fluid element relative to the total multiplicity of the event. The model samples the momenta of these balancing charges independently in the local rest frame of the fluid element (see Ref. [54] for more details.

The first step in the whole procedure was to calibrate the model without the inclusion of any CME or LCC effects, in what will be referred to in the rest of the text as "baseline". This involved tuning the input parameters to describe the centrality dependence of bulk measurements, such as the charged particle multiplicity density $d N / d \eta$ [55-57] and $v_{2}$ [58-60] in $\mathrm{Pb}-\mathrm{Pb}$ and $\mathrm{Xe}-\mathrm{Xe}$ collisions at various LHC energies. Overall the model was able to describe the experimental measurements within $15 \%$. Finally, we also checked that the slopes of the transverse momentum $\left(p_{T}\right)$ spectra of pions, kaons and protons, in the baseline sample of AVFD have a similar centrality dependence as the one reported by ALICE in Refs. [61-63].

One of the key ingredients in the development of the CME in the final state, is the early stage electromagnetic field. The AVFD model performs an event-by-event simulation of the electromagnetic field value projected along the symmetry plane, accounting for the decorrelation between the field direction and the true reaction plane due to fluctuations [43]. The initial strength of this field mainly depends on the atomic number of the nuclei that collide and the center-ofmass energy of the collision. Figure 1 presents the centrality dependence of the magnitude of $\mathbf{B}$, as simulated by AVFD at $t_{0}=0$, for $\mathrm{Pb}-\mathrm{Pb}$ and $\mathrm{Xe}-\mathrm{Xe}$ collisions at $\sqrt{s_{\mathrm{NN}}}=5.02$ and $\sqrt{s_{\mathrm{NN}}}=5.44 \mathrm{TeV}$, respectively. The values for both systems reach and for some centralities even exceed $10^{16} \mathrm{~T}$. In addition, the magnitude of $\mathbf{B}$ for a given centrality interval in collisions between $\mathrm{Pb}$-ions is larger than the corresponding value in $\mathrm{Xe}-\mathrm{Xe}$ collisions by a factor which reflects the ratio of the atomic numbers of the two nuclei. 


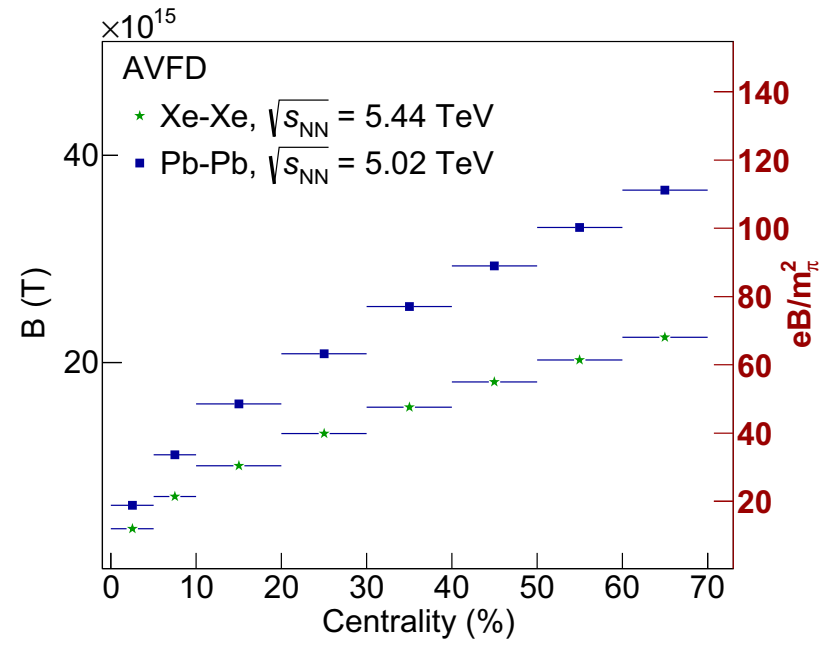

Fig. 1 The dependence of the average value of the magnetic field perpendicular to the reaction plane $\left(\mathbf{B}_{y}\right)$ on centrality for $\mathrm{Pb}-\mathrm{Pb}$ and $\mathrm{Xe}-\mathrm{Xe}$ collisions at $\sqrt{s_{\mathrm{NN}}}=5.02$ and $\sqrt{s_{\mathrm{NN}}}=5.44 \mathrm{TeV}$, respectively

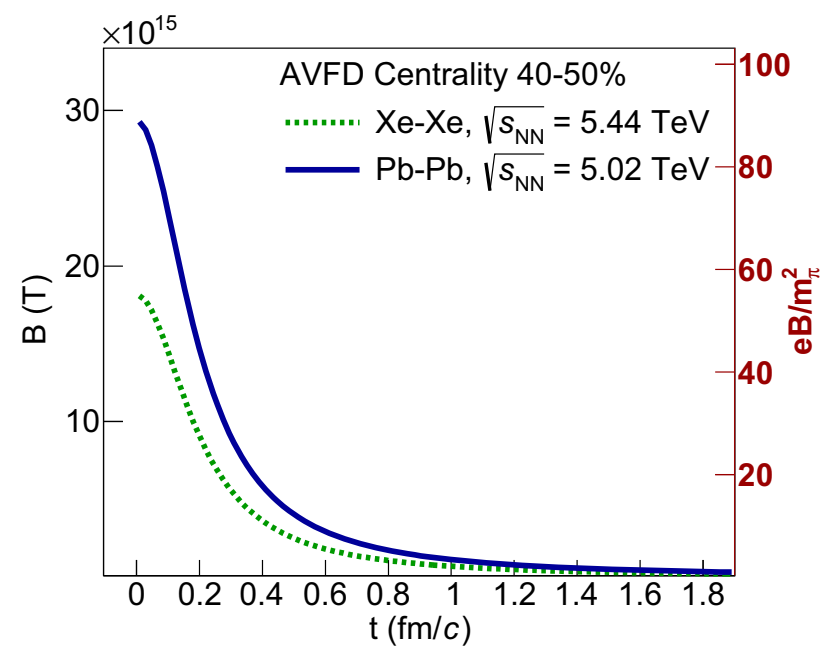

Fig. 2 The time evolution of the average value of the magnetic field perpendicular to the reaction plane (B) for the $40-50 \%$ centrality interval in $\mathrm{Pb}-\mathrm{Pb}$ and $\mathrm{Xe}-\mathrm{Xe}$ collisions at $\sqrt{s_{\mathrm{NN}}}=5.02$ and $\sqrt{s_{\mathrm{NN}}}=$ $5.44 \mathrm{TeV}$, respectively

The magnitude of the field evolves as a function of time in the model according to

$B(\tau, x)=\frac{B_{0}}{1+\tau^{2} / \tau_{B}^{2}}$,

where $\tau_{B}$ is the magnetic field lifetime which is set, in this work, conservatively to $0.2 \mathrm{fm} / c$, for both collision systems. Figure 2 presents the time evolution of the magnitude of $\mathbf{B}$ for an indicative centrality interval i.e. $40-50 \%$ for both $\mathrm{Pb}-\mathrm{Pb}$ (solid line) and Xe-Xe collisions (dashed line).

The next step in the calibration of the model required extracting the dependence of the correlators $\Delta \gamma_{1,1}$ based on Eq. 2 and $\Delta \delta_{1}$ (see Eq. 4) on both the axial current density

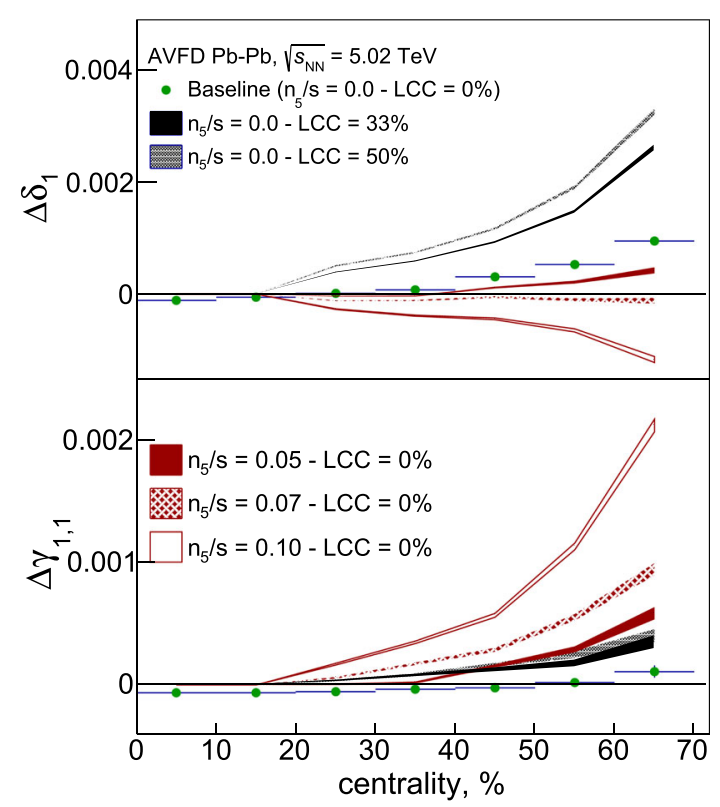

Fig. 3 The centrality dependence of $\Delta \delta_{1}$ (upper panel) and $\Delta \gamma_{1,1}$ (lower panel), the charge dependent difference of $\delta_{1}$ and $\gamma_{1,1}$ between opposite- and same-sign pairs, in $\mathrm{Pb}-\mathrm{Pb}$ collisions at $\sqrt{s_{\mathrm{NN}}}=5.02 \mathrm{TeV}$. The results from the analysis of the baseline sample are represented by the green markers. The various bands show the AVFD expectations for $\Delta \delta_{1}$ and $\Delta \gamma_{1,1}$ for various values of $n_{5} / \mathrm{s}$ (red bands) and percentage of LCC (blue bands)

$n_{5} / \mathrm{s}$ and the percentage of LCC. For this, new AVFD samples were produced for all centralities of both systems and energies, for which the amount of CME induced signal was incremented i.e., using $n_{5} / \mathrm{s}=0.05,0.07$ and 0.1 , while at the same time keeping the percentage of LCC fixed at zero. In addition, to gauge the dependence of both correlators on the background, similar number of events as before were produced where, this time, $n_{5} / \mathrm{s}$ was fixed at zero but the percentage of LCC was incremented every time. In particular, the values selected for the $\mathrm{Pb}$-system were 33 and $50 \%$. $^{1}$

Figure 3 presents the centrality dependence of $\Delta \delta_{1}$ and $\Delta \gamma_{1,1}$, in the upper and lower panels, respectively. The plots show results obtained from the analysis of events of $\mathrm{Pb}$ $\mathrm{Pb}$ collisions at $\sqrt{s_{\mathrm{NN}}}=5.02 \mathrm{TeV}$. The green markers are extracted from the analysis of the baseline sample and, in both cases, exhibit non-zero values for the majority of the centrality intervals. These non-zero values are due to the existence of hadronic resonances in the model whose decay products are affected by both radial and elliptic flow. In addition, the same plots present how the magnitude of these correlators develop for various values of the axial current density $n_{5} / \mathrm{s}$ which are represented by the red bands. It can be seen that with increasing values of $n_{5} / \mathrm{s}$ the two correlators exhibit

${ }^{1}$ Other values of LCC percentage were also checked, but due to technical reasons related to computing resources, not for all centrality intervals. 


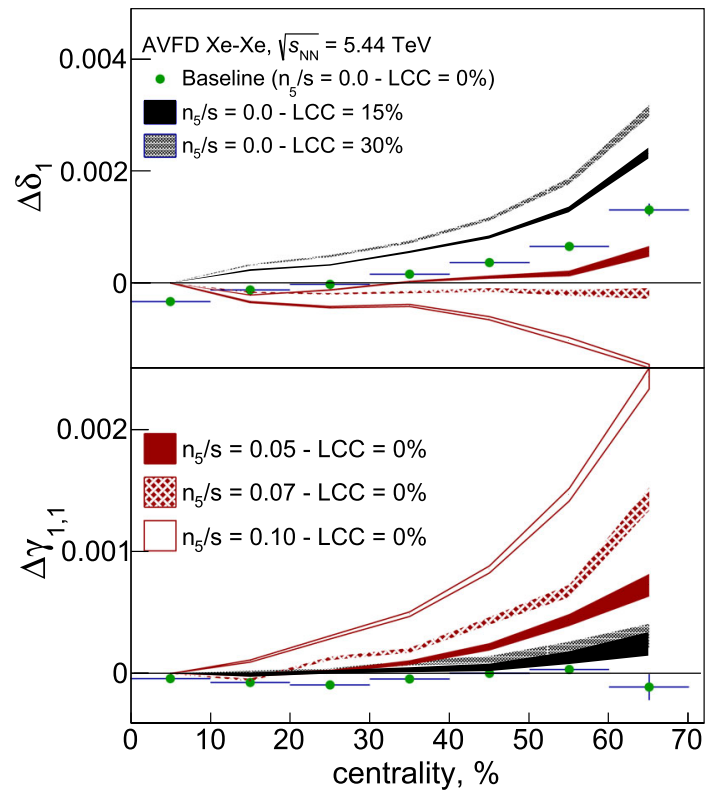

Fig. 4 The centrality dependence of $\Delta \delta_{1}$ (upper panel) and $\Delta \gamma_{1,1}$ (lower panel), the charge dependent difference of $\delta_{1}$ and $\gamma_{1,1}$ between opposite- and same-sign pairs, in Xe-Xe collisions at $\sqrt{s_{\mathrm{NN}}}=$ $5.44 \mathrm{TeV}$. The results from the analysis of the baseline sample are represented by the green markers. The various bands show the AVFD expectations for $\Delta \delta_{1}$ and $\Delta \gamma_{1,1}$ for various values of $n_{5} / \mathrm{s}$ (red bands) and percentage of LCC (blue bands)

opposite trends: while $\Delta \delta_{1}$ decreases, the values of $\Delta \gamma_{1,1}$ increase. This opposite behaviour originates from the different sign the CME contributes to $\delta_{1}$ (Eq. 4) and $\gamma_{1,1}$ (Eq. 2) and, consequently, to $\Delta \delta_{1}$ and $\Delta \gamma_{1,1}$. Finally, when fixing the value of $n_{5} / \mathrm{s}$ to zero and progressively increasing the percentage of LCC in the sample (black curves in Fig. 3), the values of both $\Delta \delta_{1}$ and $\Delta \gamma_{1,1}$ increase. However, the latter correlator exhibits a smaller sensitivity than $\Delta \delta_{1}$ to the background owning to the fact that it is constructed as the difference in the magnitude of background effects in- and out-of-plane (see Eq. 2).

Similarly, Fig. 4 presents the centrality dependence of $\Delta \delta_{1}$ and $\Delta \gamma_{1,1}$, this time in Xe-Xe collisions at $\sqrt{s_{\mathrm{NN}}}=$ $5.44 \mathrm{TeV}$. Also here the results for the baseline AVFD sample are represented with the green markers, while the red and black bands correspond to samples with progressively increasing values of $n_{5} / \mathrm{s}$ and percentage of LCC, respectively. The same qualitative observations are also found in this system: the baseline sample exhibits non-zero values for both $\Delta \delta_{1}$ and $\Delta \gamma_{1,1}$, these two correlators have opposite trends with increasing $n_{5} / \mathrm{s}$ and $\Delta \delta_{1}$ exhibits bigger sensitivity on the LCC percentage than $\Delta \gamma_{1,1}$.

To directly compare the values of $\Delta \delta_{1}$ and $\Delta \gamma_{1,1}$ between these two collision systems, the results are organised based on the input parameters used: zero $n_{5} / \mathrm{s}$ but various choices of LCC and non-zero $n_{5} / \mathrm{s}$ but LCC fixed to zero. Figures 5

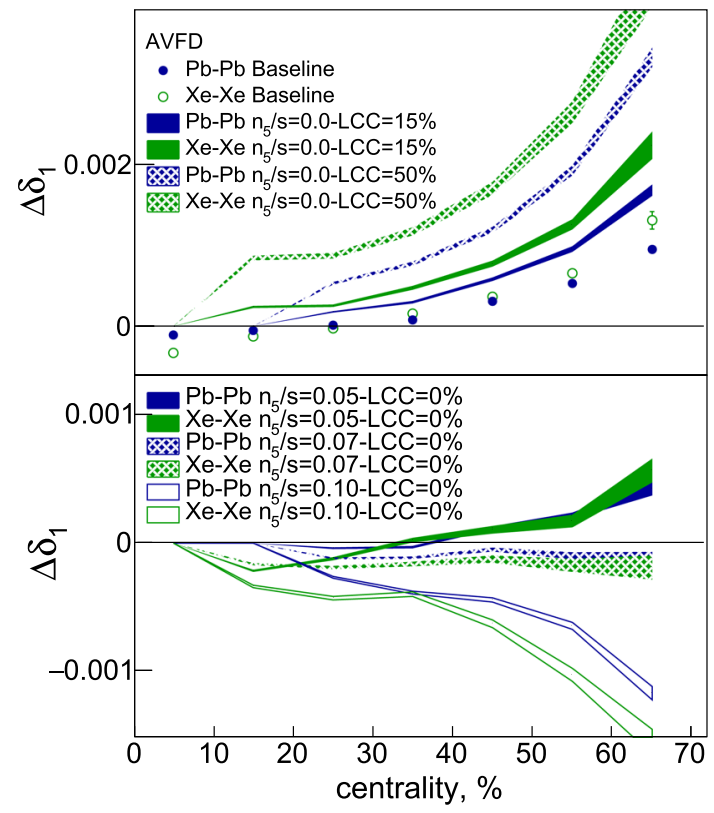

Fig. 5 The centrality dependence of $\Delta \delta_{1}$ grouped in two scenarios: zero $n_{5} / \mathrm{s}$ but various choices of LCC (upper panel) and non-zero $n_{5} / \mathrm{s}$ but LCC fixed to zero (lower panel). The various bands show the AVFD expectations for $\Delta \delta_{1}$ for $\mathrm{Pb}-\mathrm{Pb}$ collisions and $\mathrm{Xe}-\mathrm{Xe}$ collisions, with blue and green bands, respectively. The results of the baseline sample are represented by the filled and open markers



Fig. 6 The centrality dependence of $\Delta \gamma_{1,1}$ grouped in two scenarios: zero $n_{5} / \mathrm{s}$ but various choices of LCC (upper panel) and non-zero $n_{5} / \mathrm{s}$ but LCC fixed to zero (lower panel). The various bands show the AVFD expectations for $\Delta \delta_{1}$ for $\mathrm{Pb}-\mathrm{Pb}$ collisions and $\mathrm{Xe}-\mathrm{Xe}$ collisions, with blue and green bands, respectively. The results of the baseline sample are represented by the filled and open markers 
and 6, summarize the centrality dependence of the results for $\Delta \delta_{1}$ and $\Delta \gamma_{1,1}$.

In the first case, the baseline and LCC being 15 and 50\% for $\mathrm{Pb}-\mathrm{Pb}$ collisions at $\sqrt{s_{N N}}=5.02 \mathrm{TeV}$ and for $\mathrm{Xe}-\mathrm{Xe}$ collisions at $\sqrt{s_{N N}}=5.44 \mathrm{TeV}$ are chosen. The upper panel of Fig. 5 illustrates that for a fixed LCC percentage, the values of $\Delta \delta_{1}$ are higher for the $\mathrm{Xe}-\mathrm{Xe}$ than for the $\mathrm{Pb}-\mathrm{Pb}$ samples. For a fixed centrality, while the effect of radial flow between these two systems is similar [63], the charged particle multiplicity in $\mathrm{Pb}-\mathrm{Pb}$ is $60-70 \%$ higher than the corresponding value in $\mathrm{Xe}-\mathrm{Xe}$ collisions $[56,57]$. This could lead to a faster dilution of the correlations induced by the LCC mechanism in the larger system, reflected in this difference of $\Delta \delta_{1}$. At the same time, the upper panel of Fig. 6 shows that the values of $\Delta \gamma_{1,1}$ for the two systems do not exhibit any significant difference. This is in line with the expectation that the sensitivity of $\Delta \gamma_{1,1}$ to the background is significantly reduced with respect to $\Delta \delta_{1}$.

In the second case of non-zero axial current density, the samples containing $n_{5} / \mathrm{s}=0.05,0.07$ and 0.10 are chosen. The lower panel of Fig. 5 shows that $\Delta \delta_{1}$ is similar between the two systems since it is primarily affected by background contributions. This correlator needs higher values of $n_{5} / \mathrm{s}$ (e.g. $n_{5} / \mathrm{s}=0.1$ in the plot) to start observing some differences. Finally, the lower panel of Fig. 6 illustrates that the magnitude of $\Delta \gamma_{1,1}$ is higher in the $\mathrm{Xe}-\mathrm{Xe}$ than in the $\mathrm{Pb}-\mathrm{Pb}$ samples. Although the value of the magnetic field is higher for the larger Pb-system, as shown in Fig. 1, the significantly larger multiplicity that this system has, leads to a larger dilution effect reflected in the ordering of the corresponding curves in the plot.

The previous results for each colliding system and energy can be grouped in a different way that allows to parametrise the dependence of each of the correlators on the LCC percentage and on $n_{5} / \mathrm{s}$. Figures 7 and 8 present how $\Delta \delta_{1}$ and $\Delta \gamma_{1,1}$ develop as a function of the LCC percentage and $n_{5} / \mathrm{s}$, respectively. Results for the 40-50\% centrality interval of Xe-Xe collisions at $\sqrt{s_{\mathrm{NN}}}=5.44 \mathrm{TeV}$ are indicatively chosen to illustrate the procedure. An identical protocol was used for all centrality intervals of both colliding systems. One can see that both $\Delta \delta_{1}$ and $\Delta \gamma_{1,1}$ exhibit a linear dependence on the percentage of LCC, with the latter being less sensitive and thus having a smaller slope. Finally, these two correlators exhibit a quadratic dependence on $n_{5} / \mathrm{s}$ with opposite trend, originating from the dependence of $\delta_{1}$ and $\gamma_{1,1}$ on $\left\langle a_{1, \alpha} a_{1, \beta}\right\rangle$ and $-\left\langle a_{1, \alpha} a_{1, \beta}\right\rangle$ in Eqs. 4 and 2, respectively. This $a_{1}$ coefficient, in turns, has been shown in Ref. $[43,44]$ to be proportional to the value of $n_{5} / \mathrm{s}$.

Following this procedure for all centrality intervals of $\mathrm{Pb}-\mathrm{Pb}$ and $\mathrm{Xe}-\mathrm{Xe}$ collisions, one is able to parametrise the dependence of $\Delta \delta_{1}$ and $\Delta \gamma_{1,1}$ according to:

$$
\Delta \delta_{1}=c_{2} \cdot\left(n_{5} / \mathrm{s}\right)^{2}+c_{1} \cdot\left(n_{5} / \mathrm{s}\right)+b_{1} \cdot(\mathrm{LCC})+b_{0},
$$

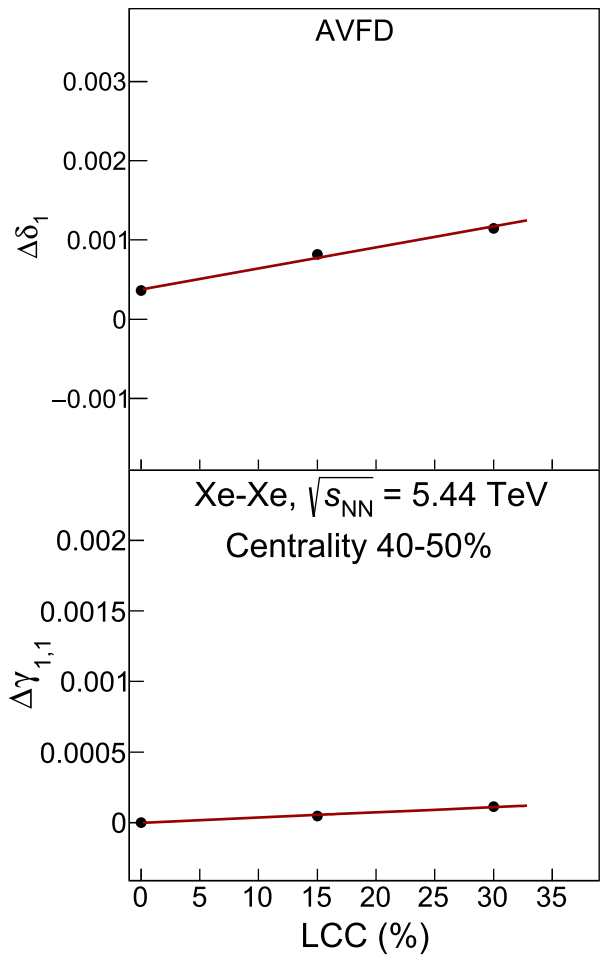

Fig. 7 The dependence of $\Delta \delta_{1}$ and $\Delta \gamma_{1,1}$ in the upper and lower panels, respectively, on the percentage of local charge conservation in the analysed samples of $\mathrm{Xe}-\mathrm{Xe}$ collisions at $\sqrt{s_{\mathrm{NN}}}=5.02 \mathrm{TeV}$ for the $40-50 \%$ centrality interval

$\Delta \gamma_{1,1}=e_{2} \cdot\left(n_{5} / \mathrm{s}\right)^{2}+e_{1} \cdot\left(n_{5} / \mathrm{s}\right)+d_{1} \cdot(\mathrm{LCC})+d_{0}$,

where $e_{2}, e_{1}, d_{1}, d_{0}, c_{2}, c_{1}, b_{1}$ and $b_{0}$ are real numbers constrained from the simultaneous fit of the corresponding dependencies of $\Delta \delta_{1}$ and $\Delta \gamma_{1,1}$ for each centrality interval of every collision system and energy. The parametrisation of Eqs. 7 and 8 assumes that the two components that control the CME signal and the background are not correlated. This is a reasonable assumption considering that the two underlying physical mechanism are independent and take place at different times in the evolution of a heavy ion collision.

\section{Results}

Having the dependence of both $\Delta \delta_{1}$ and $\Delta \gamma_{1,1}$ on $n_{5} / \mathrm{s}$ and LCC parametrised from Eqs. 7 and 8, one can deduce the values of these two parameters that govern the CME signal and the background for each centrality, colliding system and energy that allows, at the same time, for a quantitative description of the measured centrality dependence of $\Delta \delta_{1}$ and $\Delta \gamma_{1,1}$ at LHC energies.

Figure 9 presents the results of such procedure for $\mathrm{Pb}-\mathrm{Pb}$ collisions at $\sqrt{s_{\mathrm{NN}}}=5.02 \mathrm{TeV}$. The data points, extracted from Ref. [41] for both correlators are described fairly well by the tuned model. A similarly satisfactory description is 


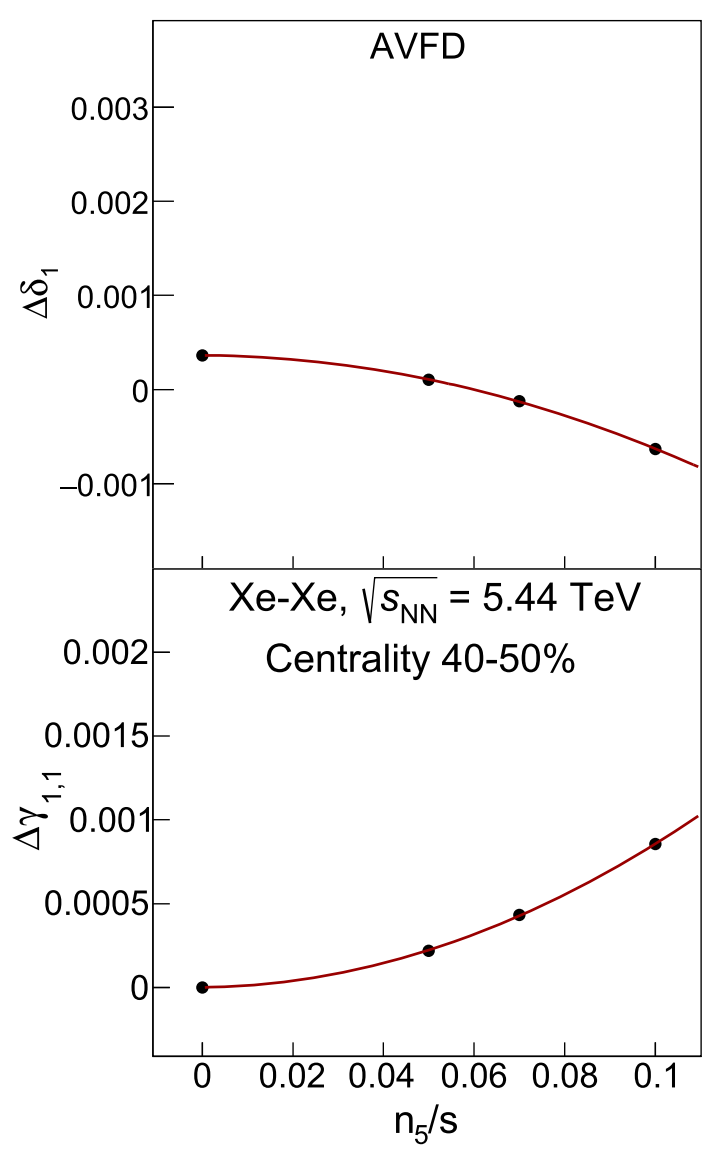

Fig. 8 The dependence of $\Delta \delta_{1}$ and $\Delta \gamma_{1,1}$ in the upper and lower panels, respectively, on the axial current density $n_{5} / \mathrm{s}$ in the analysed samples of Xe-Xe collisions at $\sqrt{s_{\mathrm{NN}}}=5.02 \mathrm{TeV}$ for the $40-50 \%$ centrality interval

also achieved for the results of Xe-Xe collisions at $\sqrt{s_{\mathrm{NN}}}=$ $5.44 \mathrm{TeV}[42]$.

Figure 10 presents the final result of the whole procedure. The plots show the centrality dependence of the pairs of LCC percentage (upper panel) and $n_{5} / \mathrm{s}$ (lower panel) that are needed to describe with AVFD the experimental measurements of $\Delta \delta_{1}$ and $\Delta \gamma_{1,1}$. The different markers represent results for different collision systems and energies. It can be seen that all systems can be described by large values of LCC that range from $40 \%$ for peripheral up to around $60 \%$ for more central $\mathrm{Pb}-\mathrm{Pb}$ collisions. There is no significant difference observed in these values among the two sets of results.

Furthermore, the lower panel of Fig. 10 illustrates that there is no significant centrality dependence of $n_{5} / \mathrm{s}$. However, there is a dependence on the colliding system. More particularly, the experimental results from the analysis of $\mathrm{Xe}-\mathrm{Xe}$ collisions lead to values of $n_{5} / \mathrm{s}$ which are compatible with zero within the uncertainties for all centrality intervals. A fit with a constant function results into values of $0.011 \pm 0.005$. This naturally results into a CME fraction which is compat-

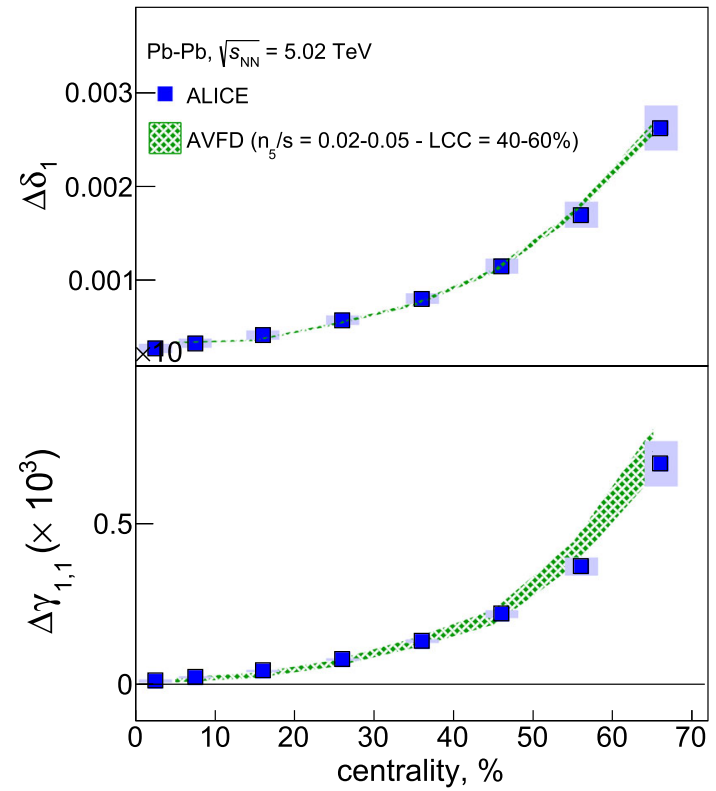

Fig. 9 The centrality dependence of $\Delta \delta_{1}$ and $\Delta \gamma_{1,1}$ in the upper and lower panels, respectively. The data points represent the experimental measurements in $\mathrm{Pb}-\mathrm{Pb}$ collisions at $\sqrt{s_{\mathrm{NN}}}=5.02 \mathrm{TeV}$ [41]. The green band shows the results obtained from the tuned AVFD sample (see text for details)

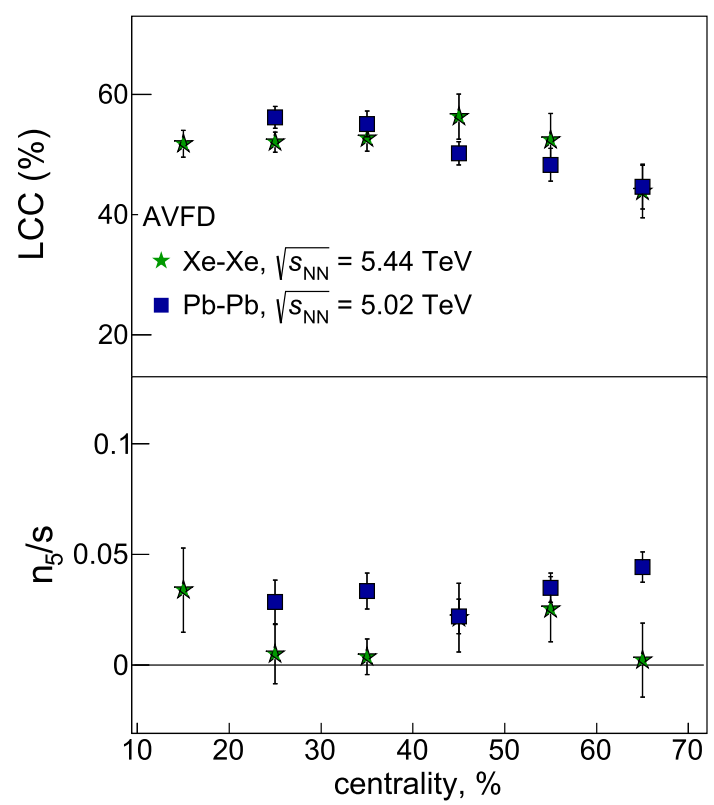

Fig. 10 The centrality dependence of the LCC percentage (upper panel) and the axial current density $n_{5} / \mathrm{s}$ that allows to describe simultaneously the experimental measurements of $\Delta \delta_{1}$ and $\Delta \gamma_{1,1}[28,41,42]$ in all collision systems and energy studied in this article

ible with zero. At the same time, the results for $\mathrm{Pb}-\mathrm{Pb}$ collisions can be described by non-zero values of axial current densities, again for the entire centrality region studied. The corresponding fit leads to a value of $0.034 \pm 0.003$ i.e., significantly above the background-only scenario. The resulting 
CME fraction gives an upper level of around 20\%, compatible with the values reported at the LHC.

\section{Summary}

In this article we presented a systematic study of charge dependent azimuthal correlations which are commonly used experimentally to probe the Chiral Magnetic Effect using the Anomalous-Viscous Fluid Dynamics framework [43,44]. After tuning the model to reproduce, within 15\%, basic experimental measurements such as the centrality dependence of the charged particle multiplicity density and the elliptic flow we were able to parametrise the dependence of both $\Delta \delta_{1}$ and $\Delta \gamma_{1,1}$ on the LCC percentage, the main contribution to the background, and the axial current density $n_{5} / \mathrm{s}$ which dictates the amount of CME signal. The resulting parametrisation is subject to uncertainties stemming from e.g. the better tuning of the baseline, the lifetime of the magnetic field which was conservatively chosen to be $0.2 \mathrm{fm} / c$ etc.

This procedure was followed for $\mathrm{Pb}-\mathrm{Pb}$ collisions at $\sqrt{s_{\mathrm{NN}}}=5.02 \mathrm{TeV}$, as well as for $\mathrm{Xe}-\mathrm{Xe}$ collisions at $\sqrt{s_{\mathrm{NN}}}=5.44 \mathrm{TeV}$. This parametrisation allowed for the estimation of the values of both the LCC percentage and $n_{5} / \mathrm{s}$ needed to describe quantitatively and at the same time the centrality dependence of $\Delta \delta_{1}$ and $\Delta \gamma_{1,1}$ measured experimentally $[28,41,42]$. The measurements in Xe-Xe are consistent with a background only scenario, with values of $n_{5} / \mathrm{s}$ compatible with zero. On the other hand, the results of $\mathrm{Pb}-\mathrm{Pb}$ collisions require $n_{5} / \mathrm{s}$ with significantly non-zero values.

Acknowledgements We are grateful to Prof. Jinfeng Liao and Dr. Shuzhe Shi for providing the source code of the model, for their guidance and their feedback during this study. We would like to thank Prof. Sergei Voloshin for his suggestions.

Data Availability Statement This manuscript has associated data in a data repository. [Authors' comment: The experimental data points used in this article can be found at https://www.hepdata.net/record/ ins 1798528 ?version $=1$. The model datasets generated and analysed during the current study are available from the corresponding author on reasonable request.]

Open Access This article is licensed under a Creative Commons Attribution 4.0 International License, which permits use, sharing, adaptation, distribution and reproduction in any medium or format, as long as you give appropriate credit to the original author(s) and the source, provide a link to the Creative Commons licence, and indicate if changes were made. The images or other third party material in this article are included in the article's Creative Commons licence, unless indicated otherwise in a credit line to the material. If material is not included in the article's Creative Commons licence and your intended use is not permitted by statutory regulation or exceeds the permitted use, you will need to obtain permission directly from the copyright holder. To view a copy of this licence, visit http://creativecomm ons.org/licenses/by/4.0/.

Funded by SCOAP ${ }^{3}$.

\section{References}

1. F. Karsch, E. Laermann, Thermodynamics and in medium hadron properties from lattice QCD. arXiv:hep-lat/0305025

2. A. Bazavov et al., Equation of state and QCD transition at finite temperature. Phys. Rev. D 80, 014504 (2009). arXiv:0903.4379 [hep-lat]

3. A. Bazavov et al., The chiral and deconfinement aspects of the QCD transition. Phys. Rev. D 85, 054503 (2012). arXiv:1111.1710 [heplat]

4. S. Borsanyi, G. Endrodi, Z. Fodor, A. Jakovac, S.D. Katz, S. Krieg, C. Ratti, K.K. Szabo, The QCD equation of state with dynamical quarks. JHEP 11, 077 (2010). arXiv:1007.2580 [hep-lat]

5. C.M.S. Collaboration, S. Chatrchyan et al., Measurement of the pseudorapidity and centrality dependence of the transverse energy density in $\mathrm{PbPb}$ collisions at $\sqrt{s_{\mathrm{NN}}}=2.76 \mathrm{TeV}$. Phys. Rev. Lett. 109, 152303 (2012). arXiv:1205.2488 [nucl-ex]

6. ALICE Collaboration, J. Adam et al., Direct photon production in $\mathrm{Pb}-\mathrm{Pb}$ collisions at $\sqrt{s_{\mathrm{NN}}}=2.76 \mathrm{TeV}$. Phys. Lett. B 754, 235-248 (2016). arXiv:1509.07324 [nucl-ex]

7. ALICE Collaboration, J. Adam et al., Measurement of transverse energy at midrapidity in $\mathrm{Pb}-\mathrm{Pb}$ collisions at $\sqrt{s_{\mathrm{NN}}}=2.76 \mathrm{TeV}$. Phys. Rev. C 94(3), 034903 (2016). arXiv:1603.04775 [nucl-ex]

8. T. Lee, A theory of spontaneous T violation. Phys. Rev. D 8, 12261239 (1973)

9. T. Lee, G. Wick, Vacuum stability and vacuum excitation in a spin 0 field theory. Phys. Rev. D 9, 2291-2316 (1974)

10. P. Morley, I. Schmidt, Strong P, CP, T violations in heavy ion collisions. Z. Phys. C 26, 627 (1985)

11. D. Kharzeev, R. Pisarski, M.H. Tytgat, Possibility of spontaneous parity violation in hot QCD. Phys. Rev. Lett. 81, 512-515 (1998). arXiv:hep-ph/9804221

12. D. Kharzeev, R.D. Pisarski, Pionic measures of parity and CP violation in high-energy nuclear collisions. Phys. Rev. D 61, 111901 (2000). arXiv:hep-ph/9906401

13. D.E. Kharzeev, Topology, magnetic field, and strongly interacting matter. Ann. Rev. Nucl. Part. Sci. 65, 193-214 (2015). arXiv:1501.01336 [hep-ph]

14. D. Kharzeev, A. Zhitnitsky, Charge separation induced by Podd bubbles in QCD matter. Nucl. Phys. A 797, 67-79 (2007). arXiv:0706.1026 [hep-ph]

15. D.E. Kharzeev, L.D. McLerran, H.J. Warringa, The effects of topological charge change in heavy ion collisions: 'event by event P and CP violation'. Nucl. Phys. A 803, 227-253 (2008). arXiv:0711.0950 [hep-ph]

16. D.E. Kharzeev, J. Liao, S.A. Voloshin, G. Wang, Chiral magnetic and vortical effects in high-energy nuclear collisions-a status report. Prog. Part. Nucl. Phys. 88, 1-28 (2016). arXiv:1511.04050 [hep-ph]

17. W. Li, G. Wang, Chiral magnetic effects in nuclear collisions. Ann. Rev. Nucl. Part. Sci. 70, 293-321 (2020). arXiv:2002.10397 [nuclex]

18. D.E. Kharzeev, J. Liao, Chiral magnetic effect reveals the topology of gauge fields in heavy-ion collisions. Nat. Rev. Phys. 3(1), 55-63 (2021). arXiv:2102.06623 [hep-ph]

19. J. Zhao, F. Wang, Experimental searches for the chiral magnetic effect in heavy-ion collisions. Prog. Part. Nucl. Phys. 107, 200-236 (2019). arXiv:1906.11413 [nucl-ex]

20. V. Skokov, A. Illarionov, V. Toneev, Estimate of the magnetic field strength in heavy-ion collisions. Int. J. Mod. Phys. A 24, 59255932 (2009). arXiv:0907.1396 [nucl-th]

21. A. Bzdak, V. Skokov, Event-by-event fluctuations of magnetic and electric fields in heavy ion collisions. Phys. Lett. B 710, 171-174 (2012). arXiv:1111.1949 [hep-ph] 
22. W.-T. Deng, X.-G. Huang, Event-by-event generation of electromagnetic fields in heavy-ion collisions. Phys. Rev. C 85, 044907 (2012). arXiv:1201.5108 [nucl-th]

23. K. Fukushima, D.E. Kharzeev, H.J. Warringa, The chiral magnetic effect. Phys. Rev. D 78, 074033 (2008). arXiv:0808.3382 [hep-ph]

24. Q. Li, D.E. Kharzeev, C. Zhang, Y. Huang, I. Pletikosic, A.V. Fedorov, R.D. Zhong, J.A. Schneeloch, G.D. Gu, T. Valla, Observation of the chiral magnetic effect in ZrTe5. Nat. Phys. 12, 550-554 (2016). arXiv:1412.6543 [cond-mat.str-el]

25. S.A. Voloshin, Parity violation in hot QCD: how to detect it. Phys. Rev. C 70, 057901 (2004). arXiv:hep-ph/0406311

26. STAR Collaboration, B.I. Abelev et al., Azimuthal charged-particle correlations and possible local strong parity violation. Phys. Rev. Lett. 103, 251601 (2009). arXiv:0909.1739 [nucl-ex]

27. STAR Collaboration, B.I. Abelev et al., Observation of chargedependent azimuthal correlations and possible local strong parity violation in heavy ion collisions. Phys. Rev. C 81, 054908 (2010). arXiv:0909.1717 [nucl-ex]

28. ALICE Collaboration, B. Abelev et al., Charge separation relative to the reaction plane in $\mathrm{Pb}-\mathrm{Pb}$ collisions at $\sqrt{s_{N N}}=2.76 \mathrm{TeV}$. Phys. Rev. Lett. 110(1), 012301 (2013). arXiv:1207.0900 [nuclex]

29. ALICE Collaboration, K. Aamodt et al., Centrality dependence of the charged-particle multiplicity density at mid-rapidity in $\mathrm{Pb}-\mathrm{Pb}$ collisions at $\sqrt{s_{N N}}=2.76 \mathrm{TeV}$. Phys. Rev. Lett. 106, 032301 (2011). arXiv:1012.1657 [nucl-ex]

30. F. Wang, Effects of cluster particle correlations on local parity violation observables. Phys. Rev. C 81, 064902 (2010). arXiv:0911.1482 [nucl-ex]

31. S. Schlichting, S. Pratt, Charge conservation at energies available at the BNL Relativistic Heavy Ion Collider and contributions to local parity violation observables. Phys. Rev. C 83, 014913 (2011). arXiv: 1009.4283 [nucl-th]

32. S. Pratt, S. Schlichting, S. Gavin, Effects of momentum conservation and flow on angular correlations at RHIC. Phys. Rev. C 84, 024909 (2011). arXiv:1011.6053 [nucl-th]

33. ALICE Collaboration, S. Acharya et al., Constraining the magnitude of the chiral magnetic effect with event shape engineering in $\mathrm{Pb}-\mathrm{Pb}$ collisions at $\sqrt{s_{\mathrm{NN}}}=2.76 \mathrm{TeV}$. Phys. Lett. B 777, 151-162 (2018). arXiv:1709.04723 [nucl-ex]

34. J. Schukraft, A. Timmins, S.A. Voloshin, Ultra-relativistic nuclear collisions: event shape engineering. Phys. Lett. B 719, 394-398 (2013). arXiv:1208.4563 [nucl-ex]

35. CMS Collaboration, A.M. Sirunyan et al., Constraints on the chiral magnetic effect using charge-dependent azimuthal correlations in $p \mathrm{~Pb}$ and $\mathrm{PbPb}$ collisions at the CERN Large Hadron Collider. Phys. Rev. C 97(4), 044912 (2018). arXiv:1708.01602 [nucl-ex]

36. STAR Collaboration, L. Adamczyk et al., Measurement of charge multiplicity asymmetry correlations in high-energy nucleusnucleus collisions at $\sqrt{s_{N N}}=200 \mathrm{GeV}$. Phys. Rev. C 89(4), 044908 (2014). arXiv:1303.0901 [nucl-ex]

37. STAR Collaboration, L. Adamczyk et al., Fluctuations of charge separation perpendicular to the event plane and local parity violation in $\sqrt{s_{N N}}=200 \mathrm{GeV} \mathrm{Au}+\mathrm{Au}$ collisions at the BNL Relativistic Heavy Ion Collider. Phys. Rev. C 88(6), 064911 (2013). arXiv: 1302.3802 [nucl-ex]

38. STAR Collaboration, L. Adamczyk et al., Beam-energy dependence of charge separation along the magnetic field in $\mathrm{Au}+\mathrm{Au}$ collisions at RHIC. Phys. Rev. Lett. 113, 052302 (2014). arXiv:1404.1433 [nucl-ex]

39. CMS Collaboration, V. Khachatryan et al., Observation of chargedependent azimuthal correlations in $p$ - $\mathrm{Pb}$ collisions and its implication for the search for the chiral magnetic effect. Phys. Rev. Lett. 118(12), 122301 (2017). arXiv:1610.00263 [nucl-ex]

40. STAR Collaboration, J. Adam et al., Charge-dependent pair correlations relative to a third particle in $p+\mathrm{Au}$ and $d+\mathrm{Au}$ collisions at
RHIC. Phys. Lett. B 798, 134975 (2019). arXiv:1906.03373 [nuclex]

41. ALICE Collaboration, S. Acharya et al., Constraining the chiral magnetic effect with charge-dependent azimuthal correlations in $\mathrm{Pb}-\mathrm{Pb}$ collisions at $\sqrt{s_{\mathrm{NN}}}=2.76$ and $5.02 \mathrm{TeV}$. JHEP 09, 160 (2020). https://doi.org/10.1007/JHEP09(2020)160. arXiv:2005.14640 [nucl-ex]

42. ALICE Collaboration, S. Aziz, Search for the chiral magnetic effect with the ALICE detector. Nucl. Phys. A 1005, 121817 (2021). arXiv:2005.06177 [hep-ex]

43. S. Shi, Y. Jiang, E. Lilleskov, J. Liao, Anomalous chiral transport in heavy ion collisions from anomalous-viscous fluid dynamics. Ann. Phys. 394, 50-72 (2018). arXiv:1711.02496 [nucl-th]

44. Y. Jiang, S. Shi, Y. Yin, J. Liao, Quantifying the chiral magnetic effect from anomalous-viscous fluid dynamics. Chin. Phys. C 42(1), 011001 (2018). arXiv:1611.04586 [nucl-th]

45. S. Shi, H. Zhang, D. Hou, J. Liao, Signatures of chiral magnetic effect in the collisions of isobars. Phys. Rev. Lett. 125, 242301 (2020). arXiv:1910.14010 [nucl-th]

46. S.A. Bass et al., Microscopic models for ultrarelativistic heavy ion collisions. Prog. Part. Nucl. Phys. 41, 255-369. arXiv:nucl-th/9803035

47. S. Voloshin, Y. Zhang, Flow study in relativistic nuclear collisions by Fourier expansion of Azimuthal particle distributions. Z. Phys. C 70, 665-672 (1996). arXiv:hep-ph/9407282

48. PHOBOS Collaboration, S. Manly et al., System size, energy and pseudorapidity dependence of directed and elliptic flow at RHIC. Nucl. Phys. A 774, 523-526 (2006). arXiv:nucl-ex/0510031

49. R.S. Bhalerao, J.-Y. Ollitrault, Eccentricity fluctuations and elliptic flow at RHIC. Phys. Lett. B 641, 260-264 (2006). arXiv:nucl-th/0607009

50. B. Alver et al., Importance of correlations and fluctuations on the initial source eccentricity in high-energy nucleus-nucleus collisions. Phys. Rev. C 77, 014906 (2008). arXiv:0711.3724 [nucl-ex]

51. B. Alver, G. Roland, Collision geometry fluctuations and triangular flow in heavy-ion collisions. Phys. Rev. C 81, 054905 (2010). arXiv:1003.0194 [nucl-th] [Erratum: Phys. Rev. C 82, 039903 (2010)]

52. B.H. Alver, C. Gombeaud, M. Luzum, J.-Y. Ollitrault, Triangular flow in hydrodynamics and transport theory. Phys. Rev. C 82 , 034913 (2010). arXiv:1007.5469 [nucl-th]

53. U. Gürsoy, D. Kharzeev, E. Marcus, K. Rajagopal, C. Shen, Chargedependent flow induced by magnetic and electric fields in heavy ion collisions. Phys. Rev. C 98(5), 055201 (2018). arXiv:1806.05288 [hep-ph]

54. S. Choudhury et al., Investigation of experimental observables in search of the chiral magnetic effect in heavy-ion collisions in the STAR experiment. arXiv:2105.06044 [nucl-ex]

55. ALICE Collaboration, E. Abbas et al., Centrality dependence of the pseudorapidity density distribution for charged particles in $\mathrm{Pb}-\mathrm{Pb}$ collisions at $\sqrt{s_{\mathrm{NN}}}=2.76 \mathrm{TeV}$. Phys. Lett. B 726, 610-622 (2013). arXiv: 1304.0347 [nucl-ex]

56. ALICE Collaboration, J. Adam et al., Centrality dependence of the charged-particle multiplicity density at midrapidity in $\mathrm{Pb}-\mathrm{Pb}$ collisions at $\sqrt{s_{\mathrm{NN}}}=5.02$ TeV. Phys. Rev. Lett. 116(22), 222302 (2016). arXiv:1512.06104 [nucl-ex]

57. ALICE Collaboration, S. Acharya et al., Centrality and pseudorapidity dependence of the charged-particle multiplicity density in Xe-Xe collisions at $\sqrt{s_{\mathrm{NN}}}=5.44 \mathrm{TeV}$. Phys. Lett. B 790, 35-48 (2019). arXiv:1805.04432 [nucl-ex]

58. ALICE Collaboration, K. Aamodt et al., Elliptic flow of charged particles in $\mathrm{Pb}-\mathrm{Pb}$ collisions at $2.76 \mathrm{TeV}$. Phys. Rev. Lett. 105, 252302 (2010). arXiv:1011.3914 [nucl-ex]

59. ALICE Collaboration, J. Adam et al., Anisotropic flow of charged particles in $\mathrm{Pb}-\mathrm{Pb}$ collisions at $\sqrt{s_{\mathrm{NN}}}=5.02 \mathrm{TeV}$. Phys. Rev. Lett. 116(13), 132302 (2016). arXiv:1602.01119 [nucl-ex] 
60. ALICE Collaboration, S. Acharya et al., Anisotropic flow in Xe-Xe collisions at $\sqrt{s_{\mathrm{NN}}}=5.44 \mathrm{TeV}$. Phys. Lett. B 784, 82-95 (2018). arXiv:1805.01832 [nucl-ex]

61. ALICE Collaboration, B. Abelev et al., Centrality dependence of $\pi, \mathrm{K}, \mathrm{p}$ production in $\mathrm{Pb}-\mathrm{Pb}$ collisions at $\sqrt{s_{N N}}=2.76 \mathrm{TeV}$. Phys. Rev. C 88, 044910 (2013). arXiv:1303.0737 [hep-ex]

62. ALICE Collaboration, S. Acharya et al., Production of charged pions, kaons and (anti-)protons in $\mathrm{Pb}-\mathrm{Pb}$ and inelastic pp collisions at $\sqrt{s_{\mathrm{NN}}}=5.02 \mathrm{TeV}$. Phys. Rev. C 101(4), 044907 (2020). https:// doi.org/10.1103/PhysRevC.101.044907. arXiv:1910.07678 [nuclex]
63. ALICE Collaboration, S. Acharya et al., Production of pions, kaons, (anti-)protons and $\phi$ mesons in Xe-Xe collisions at $\sqrt{s_{\mathrm{NN}}}=$ 5.44 TeV. Eur. Phys. J. C 81(7), 584 (2021). https://doi.org/10. 1140/epjc/s10052-021-09304-4. arXiv:2101.03100 [nucl-ex] 